\title{
Toxic Keratopathy with Bi-layer Corneal Calcific Infiltrates Caused by Proparacaine Abuse-A Case Report
}

\author{
Spencer C Cleland, Mia A Heiligenstein, Christopher M Spearman, Brendan M Lawson, Heather AD Potter, \\ and Sarah M Nehls \\ University of Wisconsin-Madison, Department of Ophthalmology and Visual Sciences, Madison, WI, USA
}

DOl: https://doi.org/10.17925/USOR.2020.13.1.40

$\mathrm{T}$ his case describes a 27-year-old man who was seen in the emergency department for an epithelial defect and subsequently developed worsening visual acuity. His vision loss was attributed to proparacaine abuse that was unresponsive to medical management. After receiving penetrating keratoplasties in both eyes, histopathology demonstrated both superficial and deep calcific infiltrates, which have not previously been described in the setting of proparacaine toxicity.

\section{Keywords}

Proparacaine, topical anesthetic, keratopathy, ring infiltrate

Disclosures: Spencer C Cleland, Mia A Heiligenstein, Christopher M Spearman, Brendan M Lawson, Heather AD Potter, and Sarah M Nehls have no financial or non-financial relationships or activities to declare in relation to this article.

Review Process: Double-blind peer review.

Compliance with Ethics: Informed consent was received from the patient involved in this case study, and no identifying information or images have been included.

Authorship: The named authors meet the International Committee of Medical Journal Editors (ICMJE) criteria for authorship of this manuscript, take responsibility for the integrity of the work as a whole, and have given final approval for the version to be published.

Access: This article is freely accessible at

touchOPHTHALMOLOGY.com

(C) Touch Medical Media 2020

Received: April 9, 2020

Accepted: May 26, 2020

Published Online: June 9, 2020

Citation: US Ophthalmic Review. 2020;13(1):40-1

Corresponding Author: Spencer C Cleland, University of Wisconsin-Madison, Department of Ophthalmology and Visual Sciences, 600 Highland Avenue, K6/448 Clinical Science Center, Madison, WI 53792, USA E: scleland0331@gmail.com

Support: No funding was received in

the publication of this article.
Proparacaine is a topical anesthetic widely used in ophthalmic practice. Proparacaine is well tolerated with limited duration of use, but has potential to become a drug of abuse. ${ }^{1}$ Extended use has numerous corneal sequelae including stromal infiltrates, corneal melt, and endothelial cell loss. ${ }^{1-3}$ In this report we present a case of proparacaine-associated keratopathy that necessitated bilateral penetrating keratoplasties with two distinct modes of corneal calcification.

\section{Case presentation}

A 27-year-old man with a history of alcohol use disorder, malnutrition, severe aplastic anemia post bone marrow transplant, and graft-versus-host disease causing chronic dry eye disease presented to the emergency department with right eye pain and light sensitivity. Visual acuity in the right eye (OD) was 20/25 and in the left eye (OS) was 20/20. Slit-lamp examination of the right cornea demonstrated an inferior epithelial defect. He was prescribed ofloxacin drops, erythromycin ointment, artificial tears, and told to follow up in clinic.

At his 1-week follow-up appointment, his visual acuity dramatically worsened to 20/125 OD and 20/150 OS. In addition, he developed large ring-shaped corneal infiltrates, filaments, and enlarged epithelial defects. Despite adding prednisolone drops and bandage contact lenses to his treatment regimen, his visual acuity remained poor at 20/100 OD and 20/400 OS 2 weeks after his initial presentation. On examination, both corneas had developed central crystalline plaques with enlarged corneal nerves (Figure 1). His clinical course and examination findings suggested a differential diagnosis including band keratopathy, peripheral ulcerative keratitis, fluoroquinolone-associated deposits, filamentary keratitis, infectious crystalline keratopathy, and neurotrophic keratitis. To address the persistent epithelial defect in both eyes, possibly attributed to neurotrophic keratitis, the patient was subsequently started on insulin drops. ${ }^{4}$

Twenty-one days following initial presentation, he was admitted to the hospital with chronic alcohol-induced pancreatitis. Upon inspection of the eye drops in his hospital room, a bottle of $0.5 \%$ proparacaine drops (Sandoz Inc., Holzkirchen, Germany) was found, which he reported having taken from the emergency room. Strict instruction to discontinue all topical anesthetic was given and a psychiatric consultation was placed. Consequently, the patient was diagnosed with major depressive disorder, anxiety, substance abuse disorder, and cluster B personality disorder.

The patient ultimately underwent penetrating keratoplasties in both eyes 4 months after his initial presentation. Histologic analysis was consistent with the slit-lamp examination, demonstrating a large central corneal ulcer and inflammation (Figure 2A). Interestingly, it also revealed both superficial and deep stromal calcium deposits, which are suggestive of concomitant band keratopathy and calcaneous degeneration (Figure 2B-C). 
Figure 1: Slit-lamp photograph of the right cornea

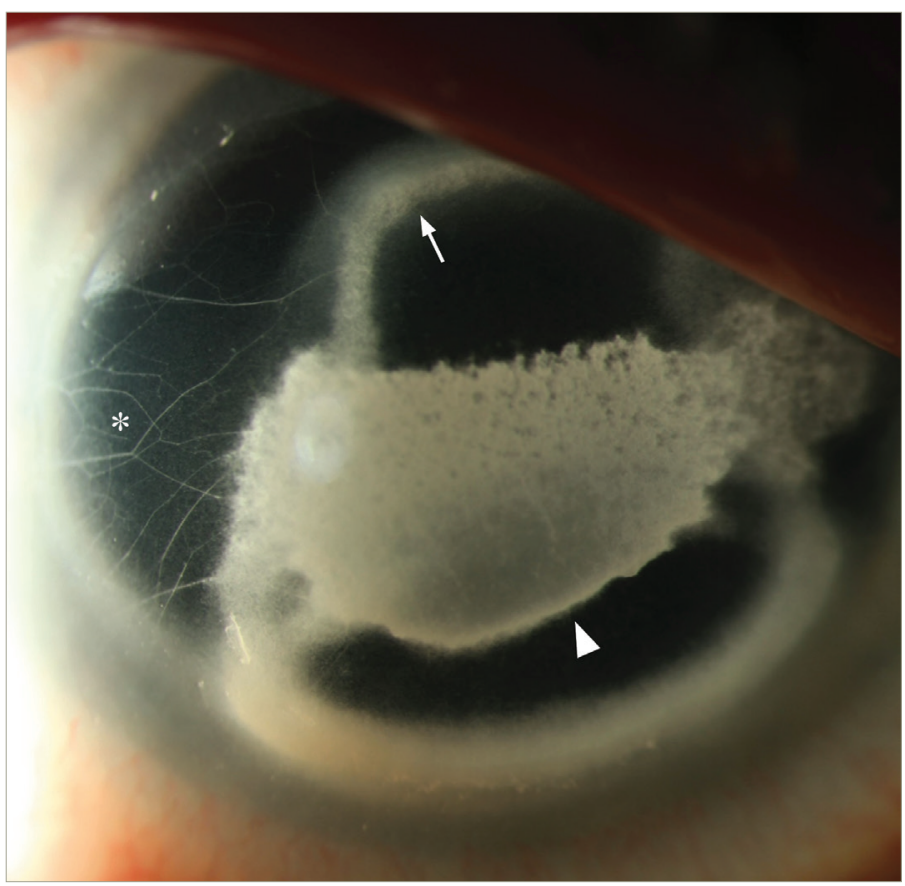

The cornea contained a large central crystalline plaque with both superficial (arrowhead) and deep (arrow) involvement, and enlarged corneal nerves (asterisk).

The patient's postoperative course was complicated by severe keratoconjunctivitis sicca secondary to graft-versus-host disease and corneal scarring of the left eye. This necessitated a repeat penetrating keratoplasty approximately 1 year later. The right corneal graft developed moderate keratic precipitates suggestive of graft rejection after 20 months. However, the keratic precipitates resolved with increased use of prednisolone drops and a subsequent taper. After 2 years of follow-up, his visual acuity was 20/40 OD and 20/20 OS.

\section{Discussion}

Topical ophthalmic anesthetics, including proparacaine and tetracaine, are safe when used appropriately to assist with examination by an ophthalmic provider. However, when abused, these drops can cause significant morbidity. Corneal toxicities include punctate epithelial erosions, persistent epithelial defects, ring-shaped stromal infiltrates, edema, Descemet membrane folds, endothelial cell loss, secondary bacterial keratitis, stromal melting, and corneal perforation..$^{1-3}$ This patient developed many of these findings within 3 weeks of use.

In addition, the patient developed bilateral calcific plaque-like infiltrates affecting both the superficial stroma and the deep stromal lamellae (Figure $2 B-C$ ). These findings correlate well to the two distinct areas of calcification seen clinically, one in which there is a dense superficial plaque and, the second, a deep stromal calcific infiltrate. The duality observed
Figure 2: Histopathologic corneal findings

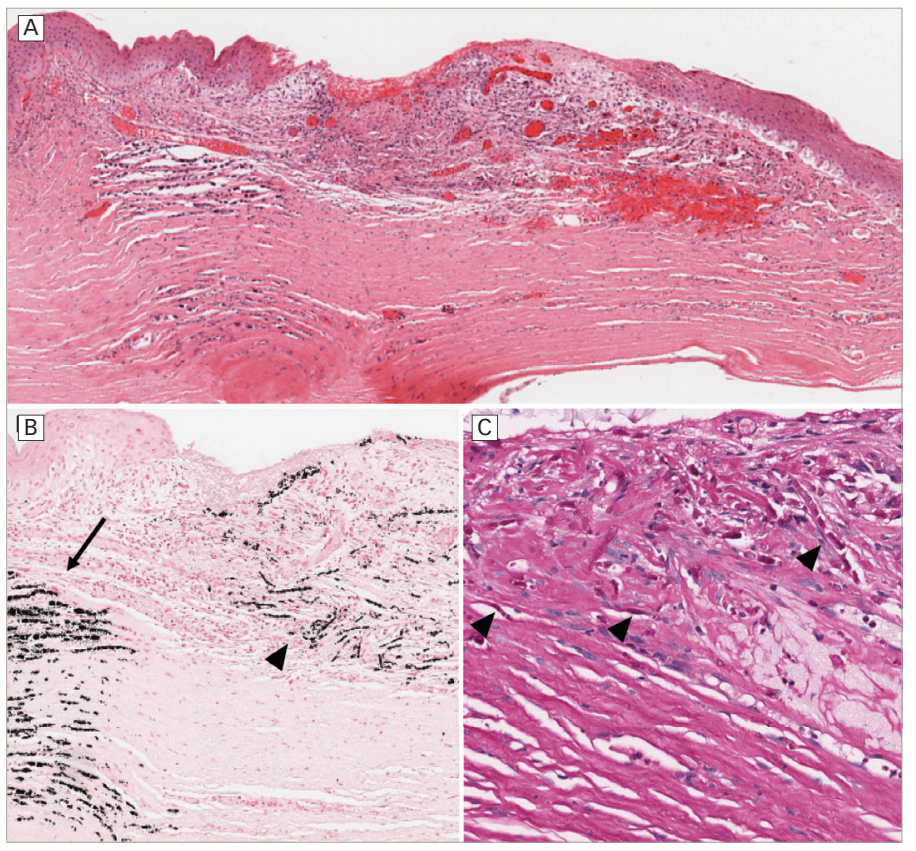

Hematoxylin and eosin stain at $4 x$ magnification of the cornea revealed central ulceration, large basophilic granular deposits within the stroma, marked corneal neovascularization, and an inflammatory infiltrate (A). Von Kossa stain at $10 \times$ magnification (B) and periodic acid-Schiff stain at 20x magnification (C) demonstrated calcium deposits within the deep lamellae (arrow) and the superficial stroma (arrowheads).

between these two patterns of calcification seen on histology is unique and has not previously been reported in association with proparacaine abuse. Band keratopathy, which can be triggered by chronic ocular inflammatory conditions and/or chemical exposures, contains calcium deposits solely within the Bowman layer and the most superficial lamellae of the stroma. ${ }^{5}$ In contrast, calcaneous degeneration of the cornea is defined by deep stromal calcifications and most commonly arises in the presence of a prolonged epithelial defect. ${ }^{6}$ We hypothesize that this patient developed concomitant band keratopathy and calcaneous corneal degeneration secondary to a persistent epithelial defect with corneal melt and proparacaine abuse.

Prevention of topical anesthetic abuse is critical for positive patient outcomes and can be significantly impacted by psychiatric disease, as seen in this patient.? Psychiatric disease can be a driving factor for continued anesthetic use, poor treatment adherence, and ultimately a poor outcome. Psychiatric comorbidities must be addressed for successful management of patients with topical anesthetic abuse.?

Topical anesthetic toxicity can be associated with poor outcomes, including loss of vision and loss of the eye. ${ }^{1-3}$ Therefore, topical anesthetics should only be used under the supervision of an ophthalmic provider and not be used outside of the clinic, emergency room, or operating room. $\square$

\footnotetext{
Yagci A, Bozkurt B, Egrilmez S, et al. Topical anesthetic abuse keratopathy: a commonly overlooked health care problem. Cornea. 2011;30:571-5

Dass BA, Soong HK, Lee B. Effects of proparacaine on actin cytoskeleton of corneal epithelium. J Ocul Pharmacol. 1988;4:187-94.

Rosenwasser GO. Complications of topical ocular anesthetics. Int Ophthalmol Clin. 1989;29:153-8.

Wang AL, Weinlander E, Metcalf BM, et al. Use of topical insulin to treat refractory neurotrophic corneal ulcers. Cornea. 2017;36:1426-8.

Jhanji V, Rapuano CJ, Vajpayee RB. Corneal calcific band keratopathy. Curr Opin Ophthalmol. 2011;22:283-9.

Lake D, Tarn A, Ayliffe W. Preservative-free eyedrops and persistent epithelial defects. 2008:27:292-6.

Tok OY, Tok L, Atay IM, et al. Toxic keratopathy associated with abuse of topical anesthetics and amniotic membrane transplantation for treatment. Int J Ophthalmol. 2015;8:938-44.
} 